\title{
Fast 3D simulation of a single-pass steel girth weld
}

\author{
E. Cottier ${ }^{1,2}$, P. Anglade ${ }^{2}$, A. Brosse ${ }^{3}$ and E. Feulvarch ${ }^{1, a}$ \\ 1 University Lyon, ENISE, LTDS, UMR 5513 CNRS, 58 rue Jean Parot, 42023 Saint-Etienne Cedex 02, France \\ 2 Haulotte Group, 27 rue d'Onzion, 42152 L'Horme, France \\ 3 ESI France, Le Recamier, 70 rue Robert, 69458 Lyon Cedex 06, France
}

Received 10 October 2014, Accepted 27 September 2015

\begin{abstract}
The prediction of welding residual stresses requires accurate account to be taken of the couplings between heat transfer, metallurgy and stresses-strains in the heat affected zone. To begin with, simulations of residual stresses for welding processes were performed at the beginning of the 1970s. Since then, calculations have been compared and validated with experimental measurements, the major problem remaining is the calculation time. Despite the technological evolution of computers, a 3D calculation can last several days. To avoid this difficulty, a 3D simplified approach is proposed in this article. It consists of decreasing the computation time for the current zone of the welds. To show the relevance of such an approach, the methodology developed is presented through the application of a single-pass steel girth weld. For this application, the computing time can be reduced by more than $67 \%$ compared to a standard "step by step" simulation.
\end{abstract}

Key words: Welding / residual stresses / finite element

\section{Introduction}

Welding has been in existence since the beginning of the 20th century and it has been widely used in many industrial fields. This assembling process induces alterations of structural and mechanical properties. Indeed the heat input causes heterogeneous cooling cycles in the material; there follow residual stresses in the heat affected zone (HAZ) and the seam weld. Measuring residual stresses inside the weld to study the propagation path of a crack, for example, is utterly impossible [1]. The numerical simulation of the welding process is therefore the only way to access the residual stress field by modeling heat transfer, metallurgy, and mechanics, as summarized in Figure 1.

For the moment, 3D numerical calculations of residual stresses are limited to several industrial fields such as the aeronautic or the nuclear industry. This is essentially due to the calculation times which can last several days. A large amount of studies have been carried out so as to decrease the computing times. For example, dynamic remeshing and later on, adaptive meshing were studied in welding simulations by Lindgren et al. [2], Runnemalm and Hyun [3], Duranton et al. [4]. Nastrom et al. [5] used a combination of solid and shell elements to model welded

\footnotetext{
${ }^{a}$ Corresponding author: eric.feulvarch@enise.fr
}

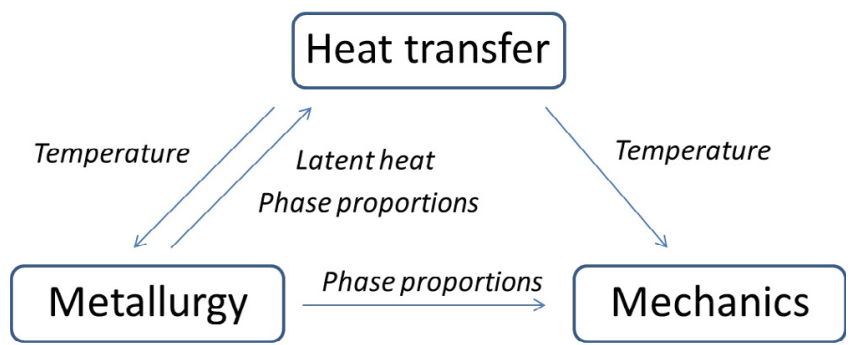

Fig. 1. Couplings between heat transfer, metallurgy and mechanics.

structures in order to reduce the number of finite elements.

Another approach, the first historically documented, consists of simplifying the problem with a 2D axisymmetric assumption. For example, Sarkani et al. [6] have shown that the thermo-mechanical 2D model of the single-pass weld of a T-joint can give fairly good results compared with those computed by a standard 3D model for the current section, except for the hoop stress which is $30 \%$ overestimated. All computations have been carried out without considering metallurgical phase changes. Another similar recent study of $\mathrm{Xu}$ et al. [7] shows that the axisymmetric 2D thermo-mechanical model of a butt-welded steel pipe leads to satisfactory results compared to the distribution of stresses obtained with a standard 3D model. 


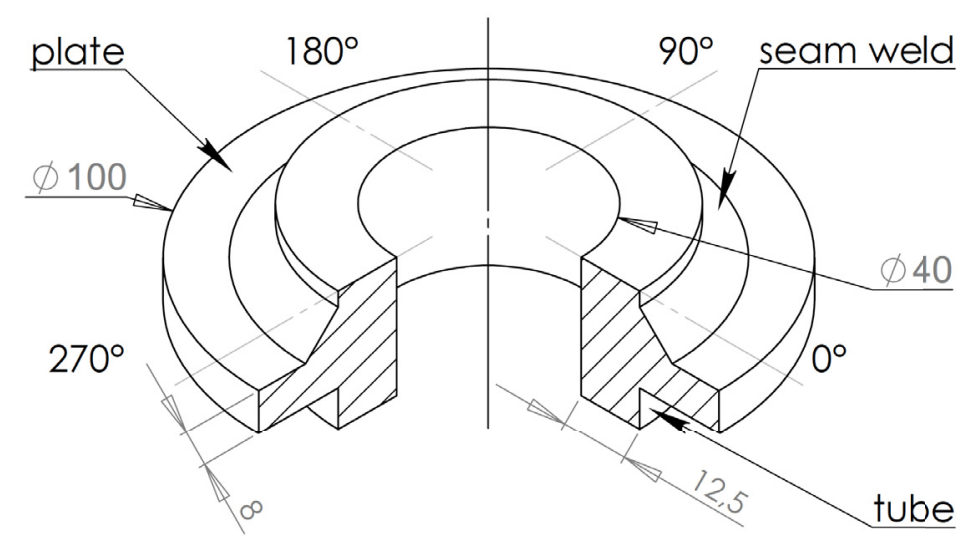

Fig. 2. Weld geometry (mm).
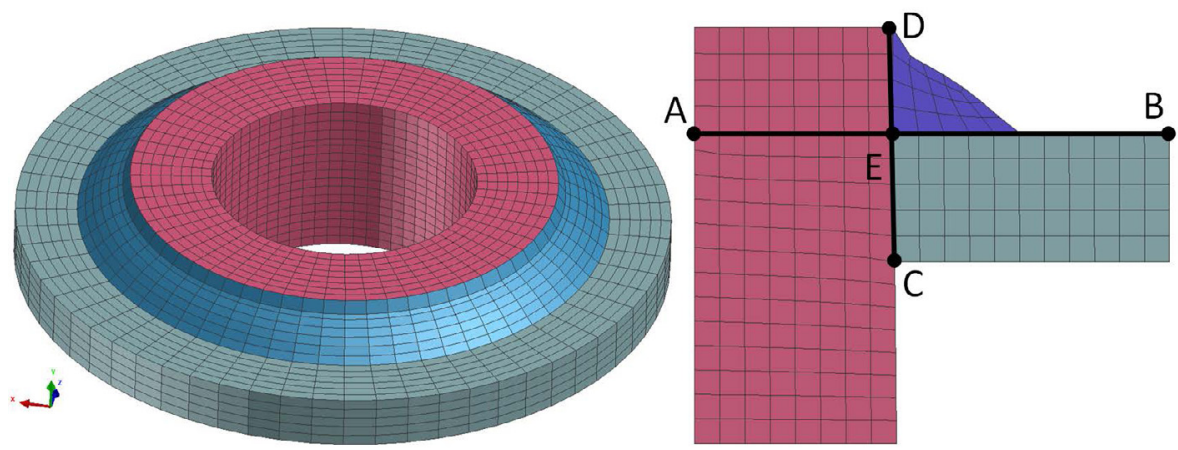

Fig. 3. 3D mesh of the weld made of 13440 tri-linear elements: longitudinal discretization of the weld (left) and discretization of a cross-section (right).

However, a significant difference in stress values can be observed for the hoop stress which is also overestimated. This is also observed by Duranton et al. [4] for a multipass weld where the metallurgical couplings have been taken into account to include tempering effects induced by each pass on the heat affected zone created by the previous passes. Tempering effects result in lowering the yield stress of the material. The consequences are very important to the residual stresses, and the temper bead welding technique is sometimes used to reduce the stress values intentionally. For a case study similar to the one of Duranton et al. [4], Feulvarch et al. [9] have shown that the 2D numerical results are in good agreement with stress measurements obtained by neutron diffraction and the deep hole drilling technique in the current region.

In this paper, we suggest a 3D simplified approach which can capture the stress distribution in the current zone but also on the start/stop regions. Given that the $2 \mathrm{D}$ assumption generally gives quite good results, the principle is to treat conventionally the start and stop zones with an equivalent $3 \mathrm{D}$ heat source and compute all the current region at the same time with an equivalent $2 \mathrm{D}$ source. The gain in computation time is thus directly related to the length of each weld and can be very significant. This methodology is presented through the application of a single-pass steel girth weld. The approach is validated in two steps for the application of a single-pass steel girth weld. First, the thermo-metallurgical results obtained are discussed according to those resulting from the standard "step by step" 3D analysis. The residual stresses are then compared showing the relevance of the simplified approach.

\section{Geometry, mesh and manufacturing process}

A T-joint links a tube with a $20 \mathrm{~mm}$ internal radius and a thickness of $12.5 \mathrm{~mm}$ with a $8 \mathrm{~mm}$ thickness plate (see Fig. 2). A hole with a $65 \mathrm{~mm}$ radius is drilled into the plate, the pipe then goes through this hole. The weld is made using the MAG (Metal Active Gas) process and a single-pass technique with a welding speed of $660 \mathrm{~mm} \cdot \mathrm{min}^{-1}$. The welding stage starts and stops at the same point corresponding to the cross-section located at the angle value of $0^{\circ}$. All components, including the filler metal, are made of ferritic steel of type $S 355$. For the computations, all the data about the ferritic steel $S 355$ are given in reference [8]. The 3D mesh used for the finite element simulations is plotted in Figure 3. The geometry of the seam weld has been design by means of a macrograph (see Fig. 4). 


\section{Computational models}

\subsection{Heat transfer}

Considering the Fourier conduction law, the heat equation writes:

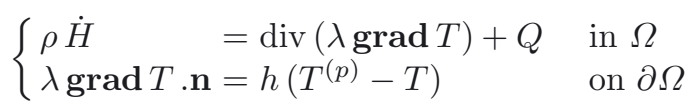

where $\rho$ is the material volumetric mass, $H$ is the specific enthalpy; $T$ is the temperature; $\lambda$ is the thermal conductivity; $T^{(p)}$ is the temperature of air and $\mathbf{n}$ is the unit outward normal vector to the boundary $\partial \Omega$ of the domain $\Omega$ representing the volume of the work pieces. $h$ is a coefficient which represents the heat exchanged between the mechanical components and the air supposed to be at the temperature $T^{(p)}$. The volume source term $Q$ involved in the heat problem corresponds to the power provided by the welding process within the material per volume unit.

The weak formulation of the heat equation is classically obtained by multiplying Equation (1) 1 by weighing function $T^{*}$ and integrating over the domain $\Omega$. Integrating by parts and accounting for the boundary condition $(1)_{2}$, one thus obtains the following weak formulation:

$$
\begin{aligned}
\int_{\Omega} \operatorname{grad} T^{*} \lambda \operatorname{grad} T \mathrm{~d} v+\int_{\Omega} T^{*} \rho \dot{H} \mathrm{~d} v \\
=\int_{\partial \Omega} T^{*} h\left(T^{(p)}-T\right) \mathrm{d} s+\int_{\Omega} T^{*} Q \mathrm{~d} v
\end{aligned}
$$

Following the usual procedure, the weak formulation (2) is applied to the finite element approximation of the function $T$ given by

$$
T \approx \sum_{i=1}^{N} N_{i} T_{i}
$$

In this expression, $N$ denotes the number of nodes, $T_{i}$ the value of the function $T$ at node $i$ and $N_{i}$ the shape function associated to this node. Following Galerkin's approach, the weighting function $T^{*}$ takes the same form.

\subsection{Metallurgical transformations and thermo-metallurgical couplings}

Three types of interaction between thermal and metallurgical phenomena must be considered [9]:

- metallurgical transformations depend on the thermal history,

- metallurgical transformations are accompanied by latent heat effects which modify temperature distributions,

- thermal properties are phase dependent.

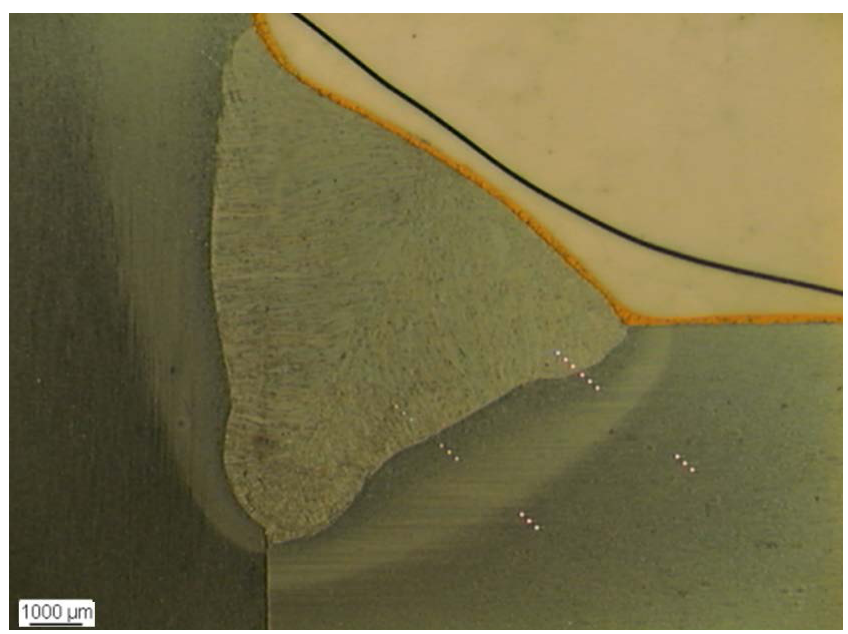

Fig. 4. Macrograph.

Different approaches can be used to describe the transformation kinetics in steels [10-12]. These approaches rest upon the modeling of transformations under either isothermal conditions (IT diagrams) or anisothermal conditions. In the latter case, parameters come from CCT diagrams. The model we use in this study belongs to the second category. Generally, those models can be expressed, in the case of one transformation, with a differential equation. Leblond and Devaux [11] have suggested the use of a simple first order differential equation of the form:

$$
\dot{p}=\frac{\bar{p}(T)-p}{\tau(T)} F(\dot{T})
$$

where $p$ is the proportion of the created phase. $\bar{p}(T)$ denotes the phase proportion obtained after an infinite time at temperature $T ; \tau(T)$ a time delay depending on temperature $T$ and $F(\dot{T})$ a function representing the dependency of the transformation rate on the temperature rate. These parameters are adjusted for each transformation in order to represent the CCT diagram. One can note that if the $F(\dot{T})$ is chosen proportional to $\dot{T}$, then the resulting phase proportion only depends on temperature as in the case for martensitic transformation which follows the Koistinen-Marburger law [13]:

$$
p(T)=\left(1-\mathrm{e}^{-b\left(M_{s}-T\right)}\right)
$$

where $M_{s}$ and $b$ respectively characterize the temperature at the beginning of transformation and the evolution of transformation with temperature. The effect of stresses on transformation kinetics has been widely examined [14], but, in practice, the lack of material data is a strong limitation to the application of models so that this effect is neglected.

In the present case study, the following metallurgical transformations are considered for the ferritic steel S355:

- initial base metal (mixture of ferrite and bainite) $\rightarrow$ austenite

- austenite $\rightarrow$ bainite 
- austenite $\rightarrow$ martensite

Once $p$ is known, the enthalpy $H$ of the mixture may be obtained from the simple "linear mixture rule":

$$
H=(1-p) H_{1}(T)+p H_{2}(T)
$$

where $H_{1}(T)$ denotes the enthalpy of the parent phase 1 , the proportion of which is $(1-p)$ and $H_{2}(T)$ that of the product phase 2 , the proportion of which is $p$. This expression disregards possible thermodynamic interactions between the phases, it is widely considered to provide an acceptable approximation of the enthalpy of alloys during phase changes. The material volumetric mass $\rho$ and the thermal conductivity $\lambda$ of the mixture may be obtained in the same way. The model has been generalized in order to consider several transformations between several phases [11]. The material thermal properties are then obtained through mixture laws between the thermal properties of all the phases.

\subsection{Mechanics}

The mechanical analysis is based on the momentum equation where inertial effects are neglected.

$$
\operatorname{div} \sigma=0 \quad \text { in } \Omega
$$

where $\boldsymbol{\sigma}$ is the Cauchy stress tensor. It is performed with the infinitesimal strain theory. Therefore, $\boldsymbol{\sigma}$ is assumed to depend linearly on the elastic strain tensor $\boldsymbol{\epsilon}^{e}$ by means of the fourth rank elastic tensor $\mathbf{C}$ as follows

$$
\boldsymbol{\sigma}=\mathbf{C}: \boldsymbol{\epsilon}^{e}
$$

As the effect of plastic dissipation on heat transfer and the influence of stresses on metallurgical transformations are neglected, the mechanical analysis can be uncoupled from the thermo-metallurgical simulation. In this way, heat transfer and metallurgy are involved in the mechanical analysis through four effects [9]:

- the thermal strains;

- the volume changes due to the transformations (contraction during heating and expansion during cooling as shown in Fig. 5);

- the influence of temperature and of the phases on the behavior law (generally, the yield strength of phases is higher if they have been created with high cooling rates);

- the transformation induced plasticity.

The strain rate tensor $\dot{\boldsymbol{\epsilon}}$ is thus decomposed into a thermal and metallurgical part $\dot{\boldsymbol{\epsilon}}^{t h}$, an elastic part $\dot{\boldsymbol{\epsilon}}^{e}$ and a plastic part $\dot{\boldsymbol{\epsilon}}^{p}$ that includes both classical plasticity and transformation induced plasticity:

$$
\dot{\boldsymbol{\epsilon}}=\dot{\boldsymbol{\epsilon}}^{t h}+\dot{\boldsymbol{\epsilon}}^{e}+\dot{\boldsymbol{\epsilon}}^{p}
$$

The volume changes due to transformations are a major cause for the appearance of residual stresses and strains. To include those changes, a simple solution consists of

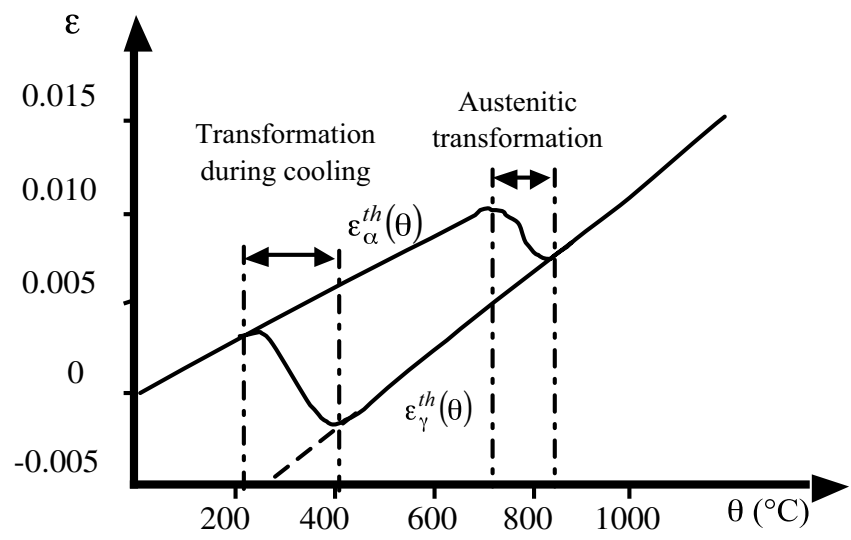

Fig. 5. Free dilatometry test.

changing the standard thermal strain into the following thermal and metallurgical strain:

$$
\boldsymbol{\epsilon}^{t h}=(1-p) \boldsymbol{\epsilon}_{1}^{t h}(T)+p \boldsymbol{\epsilon}_{2}^{t h}(T)
$$

where $\boldsymbol{\epsilon}_{1}^{\text {th }}(T)$ denotes the thermal strain of the parent phase 1 and $\epsilon_{2}^{t h}(T)$ that of the product phase 2 . $\epsilon^{t h}$ reflects both the thermal strains of the phases and the volume change between the latter as shown in Figure 5 [15]. The stress-strain relation must be temperaturedependent, representative of the phase mix, and reproduce the transformation induced plasticity phenomenon. The material behavior law during transformation is usually supposed to be elastoplastic. Plastic strain rate $\dot{\boldsymbol{\epsilon}}^{p}$ is expressed as the sum of three terms, proportional to stress variation $\dot{\boldsymbol{\sigma}}$, temperature variation $\dot{\theta}$ and phase proportion variations $\dot{p}$ respectively:

$$
\dot{\boldsymbol{\epsilon}}^{p}=a(\ldots) \dot{\boldsymbol{\sigma}}+\mathbf{b}(\ldots) \dot{\theta}+\mathbf{c}(\ldots) \dot{p}
$$

The first two terms represent the conventional plastic strain rate, while the third one represents the transformation-induced plastic strain rate. Transformation plasticity can be associated with two physical phenomena:

- Greenwood-Johnson mechanism [16]: the volume difference between the phases can generate microscopic internal stresses which are sufficient to induce plastic strain in the weaker phase (austenite).

- Magee mechanism (martensitic transformation only) [17]: in the presence of an external stress martensite needles are formed in a preferential direction.

The plasticity induced by transformation has been studied by many authors [16-19]. Different models of transformation plasticity have been proposed, but most deal with the Greenwood-Johnson mechanism only. Among all the models related to the behavior of steels during phase transformation, the model proposed by Leblond and coworkers [20], based on a micro-mechanical analysis, is widely used. The model requires the temperature dependent stress-strain relations of all the phases which 
is quite difficult to obtain [21]. The hardening can be isotropic or kinematic, and viscoplastic effects can also be considered [22]. In the present study, the behavior of the different constitutive phases of $S 355$ steel is assumed to be elastoplastic with isotropic hardening. All details on the elastoplastic behavior including the mixed phase relations used in this work are detailed by Devaux et al. [23].

To apply the finite element method, the weak formulation for the mechanical problem is obtained by multiplying Equation (7) by weighing function $\boldsymbol{U}^{*}$ and integrating over the domain $\Omega$. The boundary conditions can be prescribed displacements or stress vectors imposed. Integrating by parts and considering that the surfaces of the mechanical components are stress free, one thus gets:

$$
\begin{aligned}
\int_{\Omega} \boldsymbol{\epsilon}\left(\boldsymbol{U}^{*}\right): \boldsymbol{\sigma}(\boldsymbol{U}) \mathrm{d} v & =0 \quad \text { with } \boldsymbol{\epsilon}\left(\boldsymbol{U}^{*}\right) \\
& =\frac{1}{2}\left(\operatorname{grad} \boldsymbol{U}^{*}+\operatorname{grad}^{t} \boldsymbol{U}^{*}\right)
\end{aligned}
$$

Following the usual procedure, the weak formulation (12) is applied to the finite element approximation of the displacement $\boldsymbol{U}$ which is similar to the one used for the temperature approximation:

$$
\boldsymbol{U} \approx \sum_{i=1}^{N} N_{i} \boldsymbol{U}_{i}
$$

\section{Thermal simulation}

As mentioned in the previous section, the internal heating due to plastic dissipation is always neglected considering the small strain rates generated by a welding operation [24]. Therefore, the simulation can be achieved through a staggered method which consists of splitting the analysis into two steps. First, the temperature and phase variations are determined as a function of time. Then, the mechanical computation uses the previous results to get displacements, stresses and distortions.

\subsection{Equivalent heat source modeling}

To compute the thermal cycles, several input data are necessary: the geometries of the different parts, the material properties and the equivalent heat source. Some methods are proposed in literature in order to model the heat input. For example, Goldak et al. [25] propose a two half-ellipsoid heat source shape which needs to be readjusted by means of multiple coefficients. This method is well adapted to processes with filling materials. The readjustment can take place using thermocouple sensors to measure the temperature at different locations but this method cannot always capture the fast temperature changes near the welding pool because of stability problems [7]. A second approach consists of preparing the macrograph of a cross-section of the weld; and fitting heat source parameters to make sure that simulated geometric

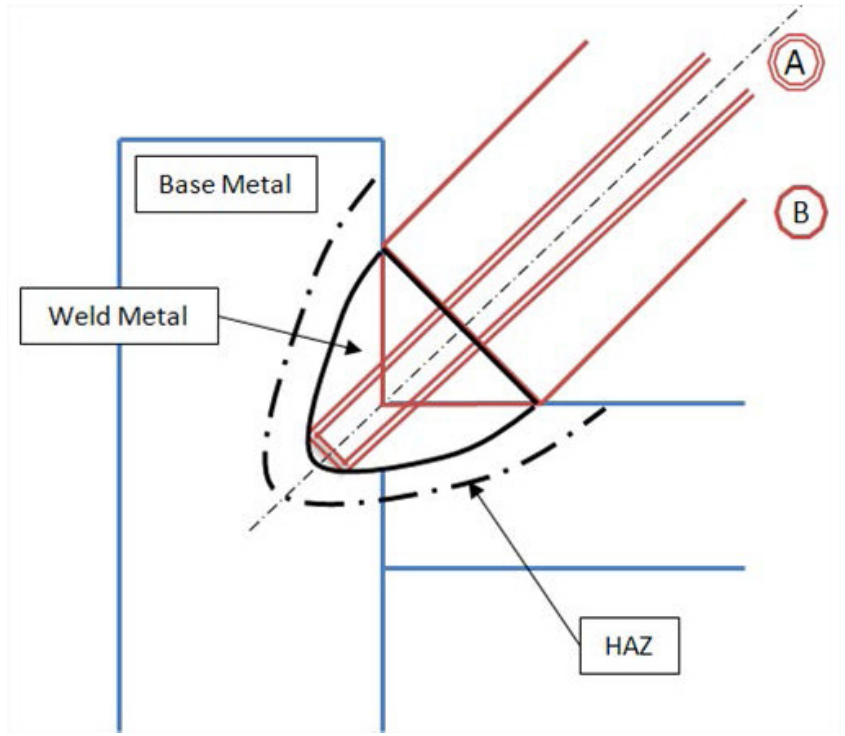

Fig. 6. Schematic of the heat input model in a cross section.

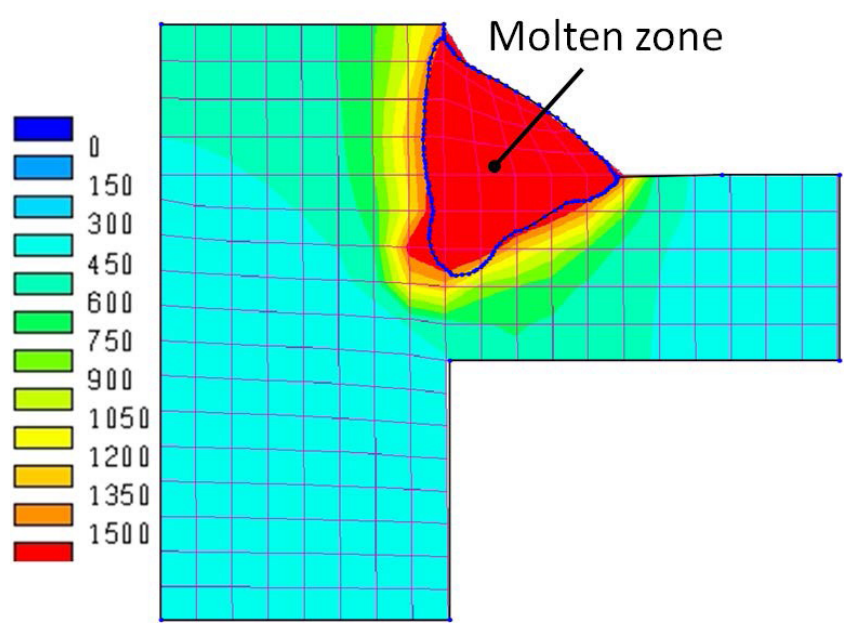

Fig. 7. Comparison between the macrograph and the distribution of maximum computed temperatures $\left({ }^{\circ} \mathrm{C}\right)$ at $180^{\circ}$.

appearance of and the molten zone matches the geometric dimensions observed on the weld macrograph as shown in Figure 4.

In this study, we have chosen the second method for its simplicity by considering a simple heat source shape plotted in Figure 6. The heat input is represented by two cylinders of power per volume unit $Q(\mathbf{x}, t)$ (see Eq. (1)): a small cylinder of high power (Fig. 6A) in order to simulate the penetration observed on the macrograph and a second bigger one of lower power (Fig. 6B) for modeling the shape of the molten zone. Figure 7 shows the comparison between the molten zone identified on the macrograph, and the distribution of maximum computed temperatures on the cross-section located at an angle of $180^{\circ}$ in the current region (Fig. 2). The tridimensional simulation is carried out on the whole geometry of the weld using the $3 \mathrm{D}$ mesh plotted in Figure 3 and a standard implicit step 


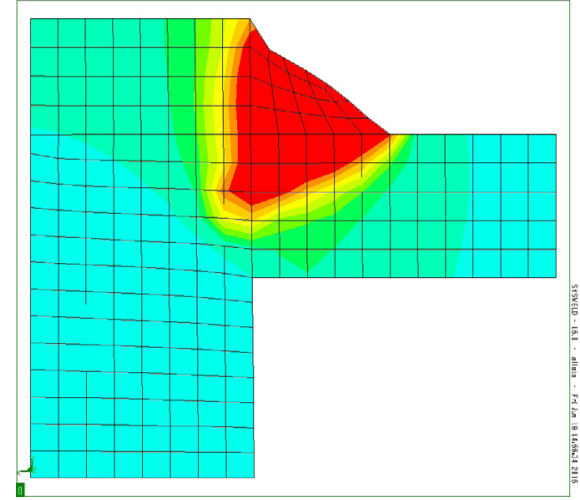

(a) $3 \mathrm{D}$

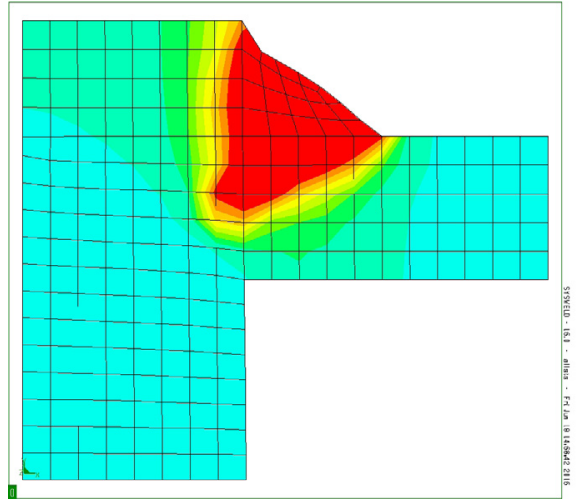

(c) $2 \mathrm{D}$

Fig. 8. Distribution of the maximum temperatures $\left({ }^{\circ} \mathrm{C}\right)$ in $3 \mathrm{D}$ at $180^{\circ}$ (left) and in $2 \mathrm{D}$ (right).

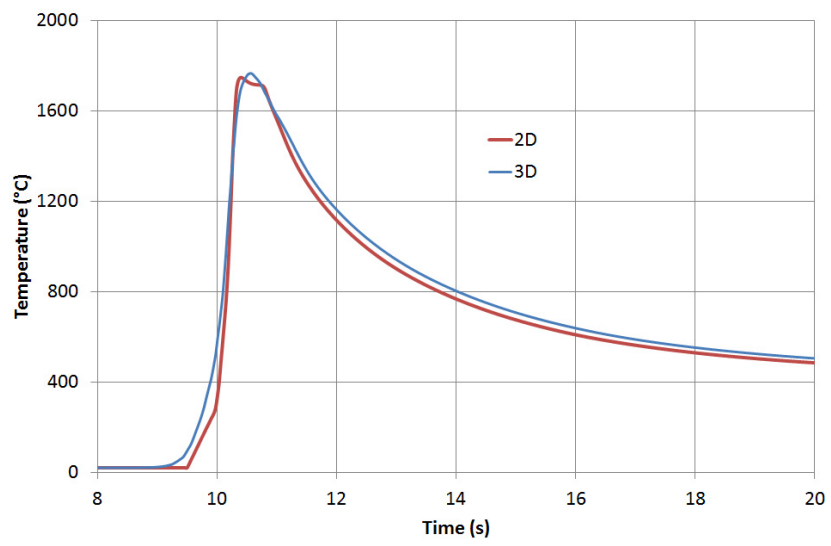

Fig. 9. Comparison of the temperature evolutions at node $E$ in $3 \mathrm{D}$ at $180^{\circ}$ and in $2 \mathrm{D}$ (see Fig. 3).

by step approach. In this paper, all the simulations have been performed using the computer code Sysweld ${ }^{\circledR}[8]$.

\subsection{D simplified approach}

As mentioned in the introduction, many authors have shown that results obtained with a standard 3D FEM approach can be approximated in the current region by means of $2 \mathrm{D}$ axisymmetric modeling. This configuration is less time consuming and less complicated. It corresponds to the assumption that all the filling material is deposited at the same time. Unfortunately, the 3D equivalent heat source can not be directly implemented in the $2 \mathrm{D}$ simulation, because the associated heat transfers are also 3D.

Let $Q(\mathbf{x}, t)$ be the power per volume unit representing the $3 \mathrm{D}$ equivalent heat source defined in the previous subsection; Sarkani et al. [6] have shown that a 2D equivalent heat source can be obtained by multipying the $3 \mathrm{D}$ source by a coefficient $\gamma$. Let us consider a cross-section, the authors explained that the coefficient $\gamma$ for this section is due to the power transferred by the neighbour cross-section where the welding torch is located before its arrival. In this work, this coefficient has been fitted using

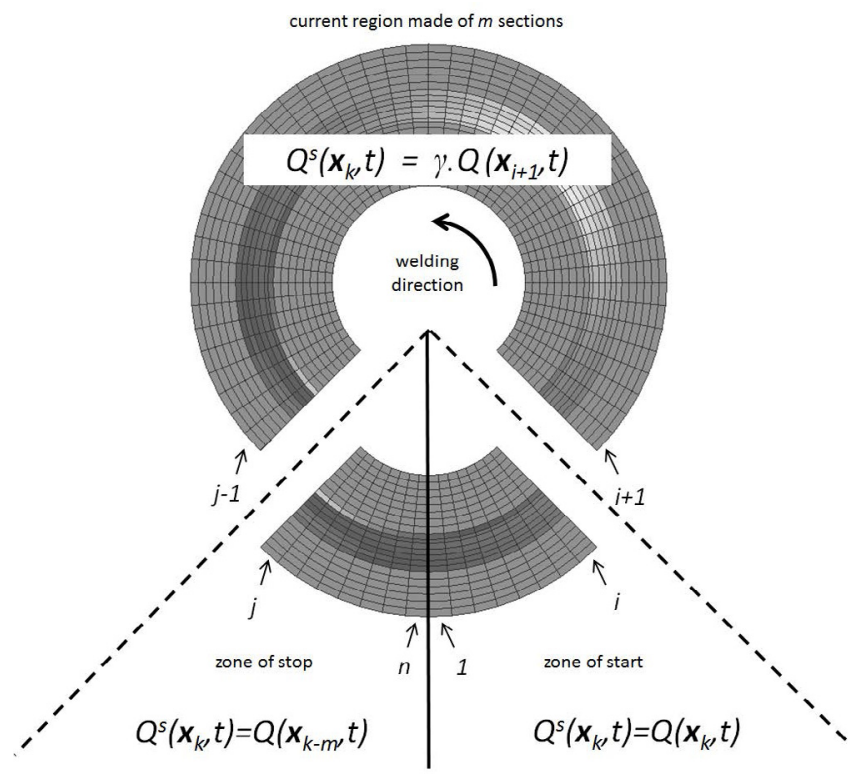

Fig. 10. Schematic of the simplified equivalent heat source for a weld made of $n$ sections.

a 2D axisymmetric model based on the $2 \mathrm{D}$ mesh plotted in Figure 3. The value of $\gamma$ has been obtained by comparisons of the maximum temperatures (see Fig. 8). It is taken equal to 0.96. As shown in Figure 9, the temperature evolutions are very close. Unfortunately, 2D modeling does not allow to simulate heat transfer hence residual stresses in the start and stop zones.

The principle of the technique developed in this paper is to use the 3D equivalent heat source for the start and stop areas and the $2 \mathrm{D}$ equivalent heat source for the current region as shown in Figure 10. Let $Q^{s}(\mathbf{x}, t)$ be the new 3D heat source; the simplified 3D approach consists of replacing $Q(\mathbf{x}, t)$ by $Q^{s}(\mathbf{x}, t)$ in Equation (1). Considering a weld composed of $n$ sections with a current region containing $m$ sections, the position of each section $k$ is denoted $\mathbf{x}_{k}$. As shown in Figure 10, the new heat source is identical to the initial equivalent heat source in the start zone. For the current region, the heat input is the same 


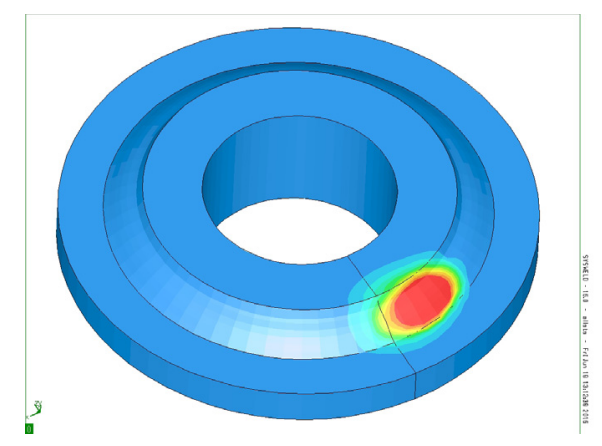

(a) $\mathrm{t}=1.03 \mathrm{~s}\left(\theta=22.5^{\circ}\right)$

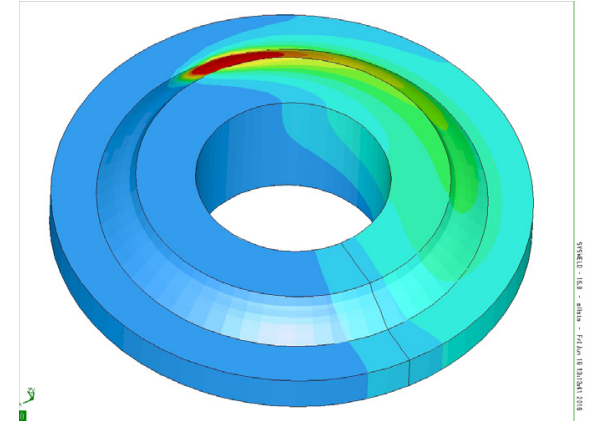

(b) $\mathrm{t}=10.17 \mathrm{~s}\left(\theta=180^{\circ}\right)$
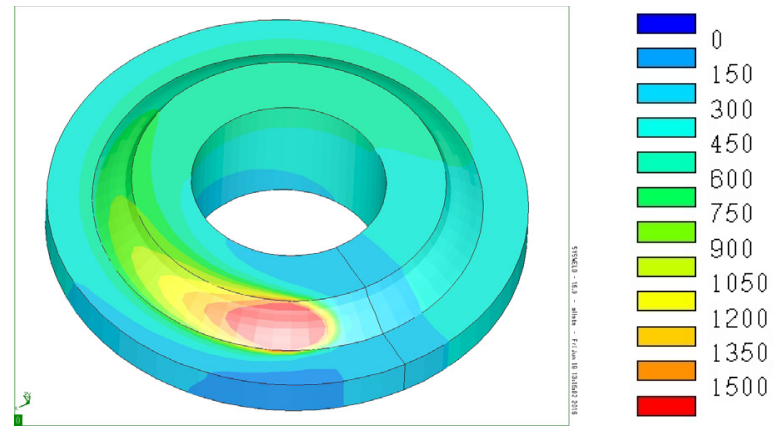

(c) $\mathrm{t}=19.07 \mathrm{~s}\left(\theta=337.5^{\circ}\right)$

Fig. 11. Distribution of temperature $\left({ }^{\circ} \mathrm{C}\right)$ for the standard step by step simulation (case a).

in all sections. It is the same as the one received by the section numbered $i+1$ for a standard simulation, multiplied by the coefficient $\gamma \cdot \gamma$ is necessary because there is very little heat transfer from one section to another in the current region. When the heating starts in the current region, at the same time, the section $j$ receives a heat input identical to the one of the section numbered $i+1$ for a standard simulation. In this way, the time for the heating of the current region does not explicitly appear in the simulation. Then, the source continues its path along the welded joint in the stop area. As a result, the gain of computing time can be approximated by the ratio $m / n$ for the proposed case study.

Figures 11-13 show the change in temperature for different position of the heat source. The first position corresponds to an angle of $22.5^{\circ}$ of the axis of the heat source. This angle is common to the three simulations since it is upstream of the welding current region. The second position corresponds to an angle of $180^{\circ}$ for the step by step simulation. For the simplified simulations, the second angle is the one of the beginning of the current region. The third position is out of the current region just before coming back to the start point. One can note that for the third case (see Fig. 13), the start area has not enough time to cool down and the temperature is still very important. This can be observed in Figure 14.

A comparison of the distribution of the maximum temperatures is plotted in Figure 15 for the standard simulation (case a) and the new simplified method with 2 lengths of the current zone: $90^{\circ} \rightarrow 270^{\circ}$ (case b) and $45^{\circ} \rightarrow 315^{\circ}$ (case c). One can note that the results obtained with the simplified approach are in good agreement with the ones of the standard simulation. As shown in Figure 16, the temperature evolutions are again very close to the standard simulation. In Figure 16, the time has been readjusted to correspond to an identical instant of heat input through the cross-section located at $180^{\circ}$.

\section{Mechanical analysis}

Once the thermo-metallurgical simulation has been carried out, the mechanical computation uses the temperature and phase distribution in order to get displacements and stresses. For practical reasons, the mesh used for the thermo-metallurgical computation is also the one used for the 3D mechanical simulation. This approach avoids the problem of the internal variables projection from one mesh to another. The finite elements corresponding to the filling material are mechanically activated under the solidus temperature to model the addition of material according to the welding thermal kinetics [9]. Figure 17 shows the distribution of the von Mises residual stresses for the 3 thermo-metallurgical simulations developed in the previous section.

As shown in Figures 17 and 18, a slight difference in the current region appears between the reference (a) and the simplified simulation (b).

This can be explained by the fact that the weld is relatively short and that the calculation (a) does not exhibit a real steady state corresponding to the current region. Indeed, the hypothesis of a current region appears clearly 
E. Cottier et al.: Mechanics \& Industry 17, 401 (2016)

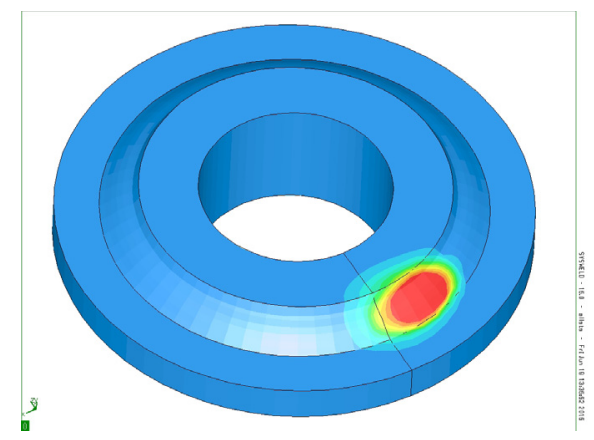

(a) $\mathrm{t}=1.03 \mathrm{~s}\left(\theta=22.5^{\circ}\right)$

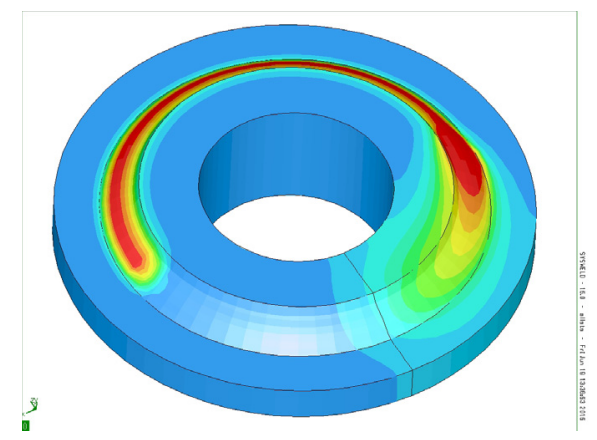

(b) $\mathrm{t}=5.08 \mathrm{~s}\left(\theta=90^{\circ}\right)$
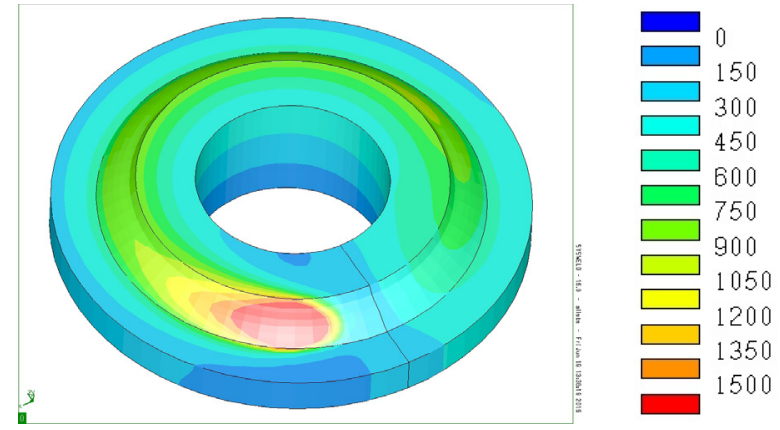

(c) $\mathrm{t}=8.9 \mathrm{~s}\left(\theta=337.5^{\circ}\right)$

Fig. 12. Distribution of temperature $\left({ }^{\circ} \mathrm{C}\right.$ ) for the simplified simulation with a current zone going from $90^{\circ}$ to $270^{\circ}$ (case b).

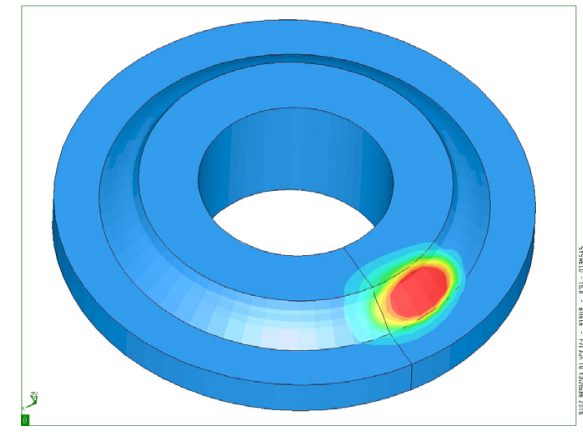

(a) $\mathrm{t}=1.03 \mathrm{~s}\left(\theta=22.5^{\circ}\right)$

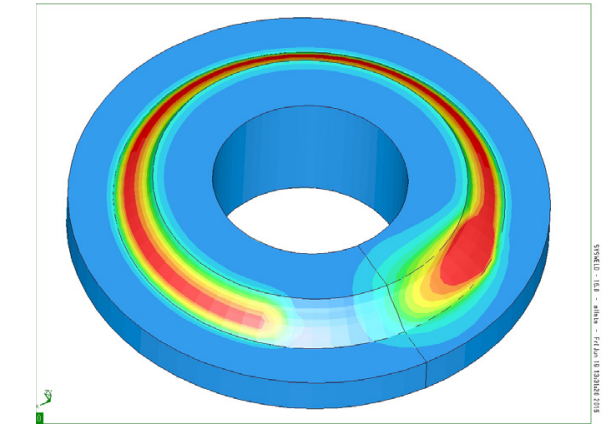

(b) $\mathrm{t}=2.54 \mathrm{~s}\left(\theta=45^{\circ}\right)$
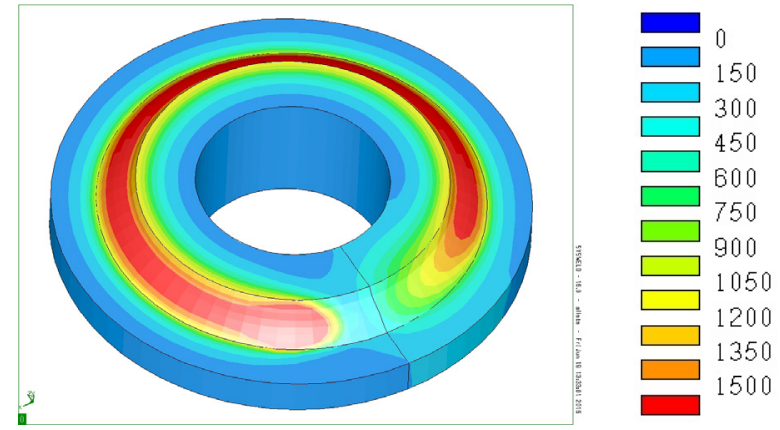

(c) $\mathrm{t}=3.81 \mathrm{~s}\left(\theta=337.5^{\circ}\right)$

Fig. 13. Distribution of temperature $\left({ }^{\circ} \mathrm{C}\right.$ ) for the simplified simulation with a current zone going from $45^{\circ}$ to $315^{\circ}$ (case c). 


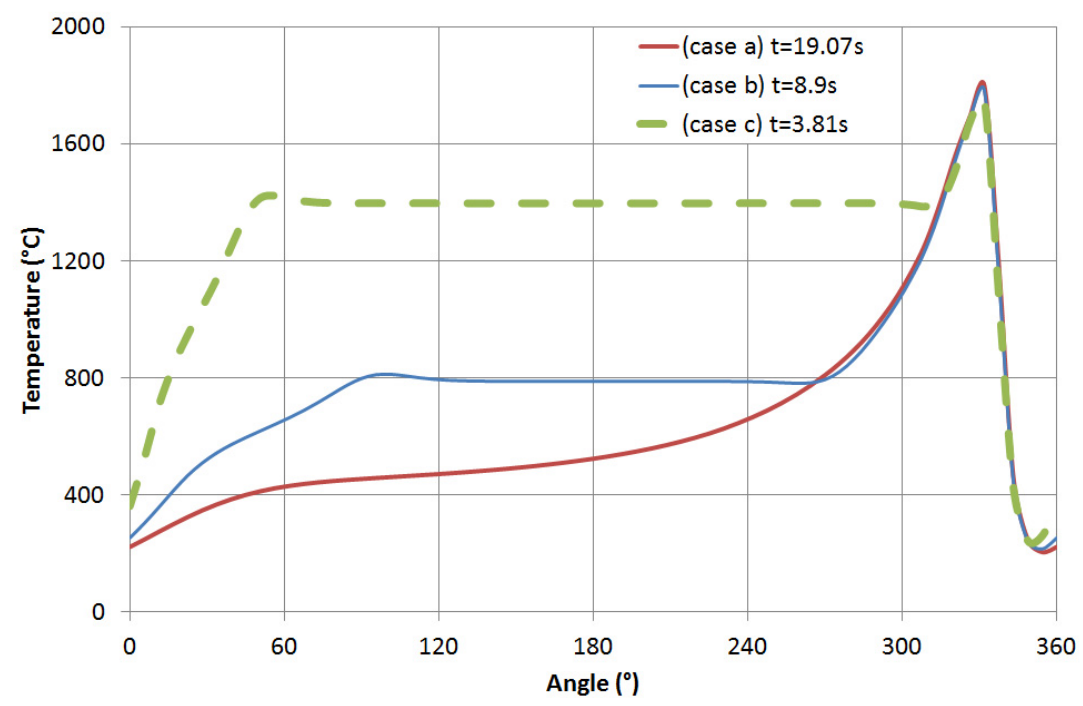

Fig. 14. Distribution of the temperature at node $E$ (see Fig. 3).

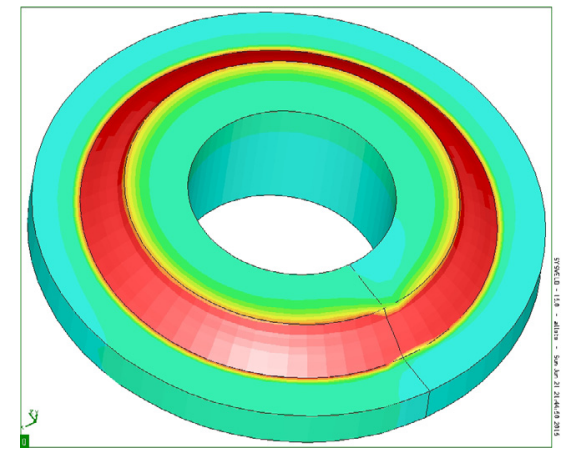

(a) case a

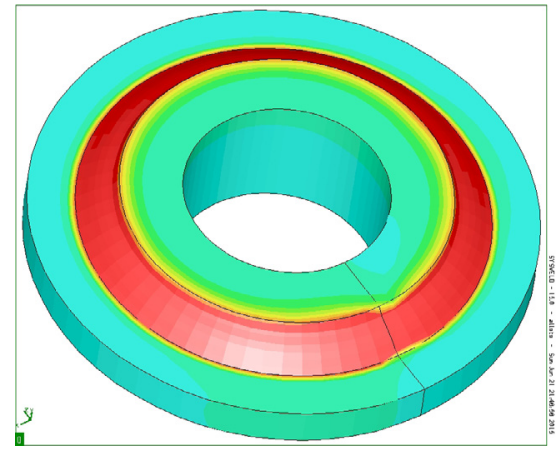

(b) case b

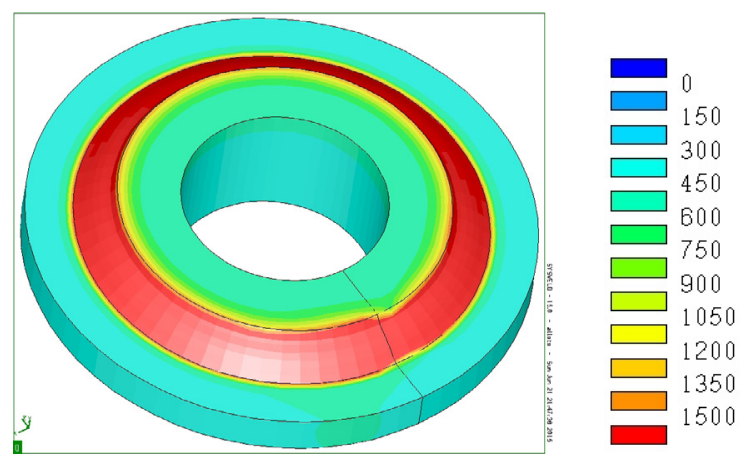

(c) case $\mathrm{c}$

Fig. 15. Distribution of the maximum temperatures $\left({ }^{\circ} \mathrm{C}\right)$ for the standard simulation (a) and 2 simplified simulations with 2 lengths of the current zone: $90^{\circ} \rightarrow 270^{\circ}$ (b) and $45^{\circ} \rightarrow 315^{\circ}$ (c).

for case $(\mathrm{b})$ on half the welding $\left(90^{\circ} \rightarrow 270^{\circ}\right)$. The difference with the standard simulation will be mitigated in the case of longer welds. The difference appears more clearly with case $\mathrm{c}$. This is explained by the fact that the starting and stopping areas are halved compared to case b. This last case shows that the lengths of the start and stop zones must be chosen carefully in order to obtain satisfactory residual stresses in the whole weld. As shown in
Figure 14, the start area in case (c) has not enough time to cool down as quickly as for cases (a) and (b). Therefore, the thermo-metallurgical state of the start zone differs significantly compared to the reference simulation (a) when the heat source reaches the end of the welding sequence. To obtain residual stresses similar to the reference simulation (a) near the start point, it would be appropriate to lengthen the start region up to $90^{\circ}$ as for case (b) to 
E. Cottier et al.: Mechanics \& Industry 17, 401 (2016)

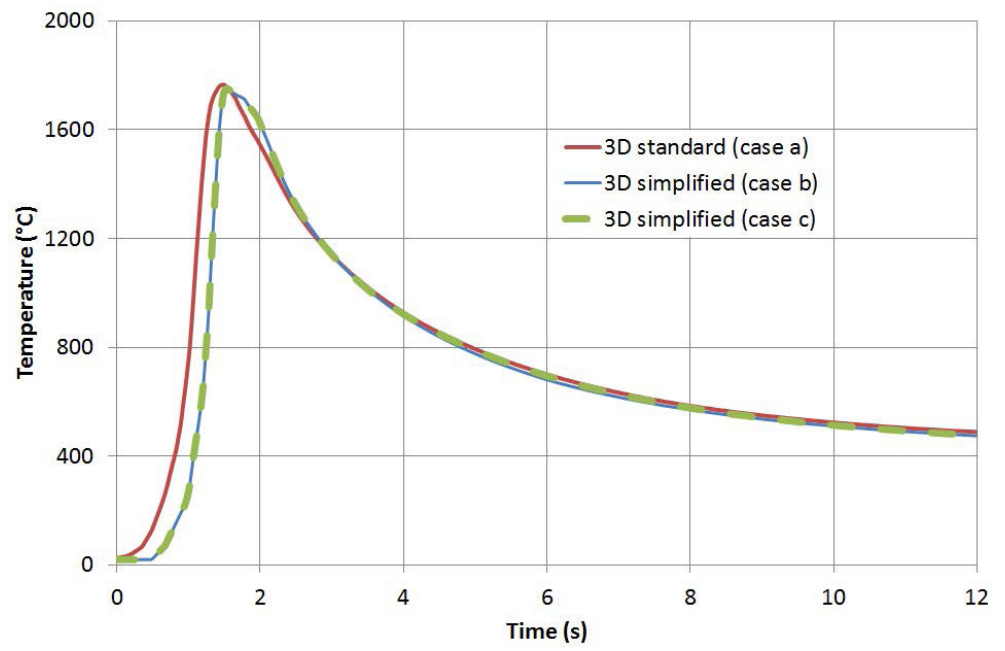

Fig. 16. Comparison of the temperature evolutions at node $E$ in the section located at $180^{\circ}$ (see Fig. 3).

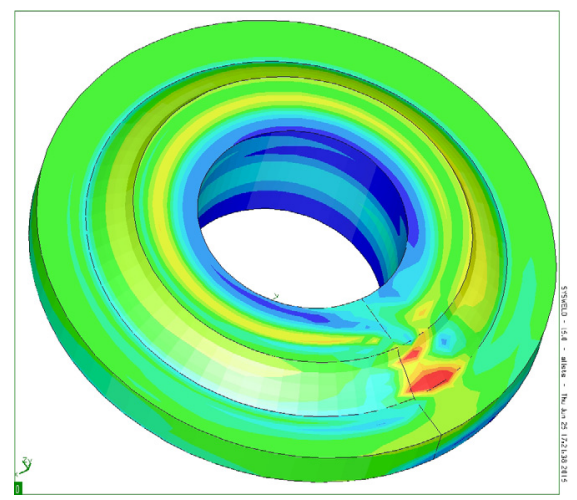

(a)

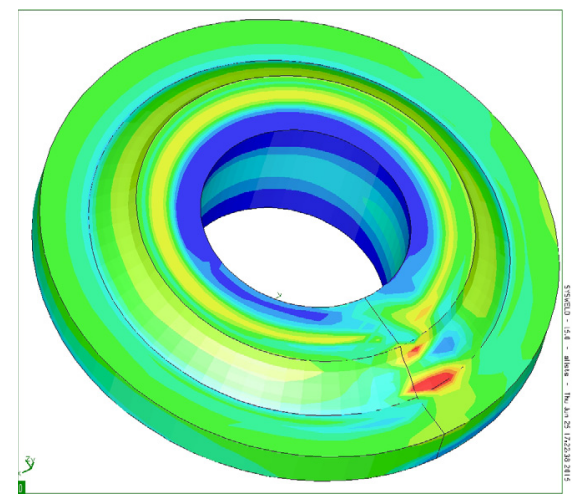

(b)
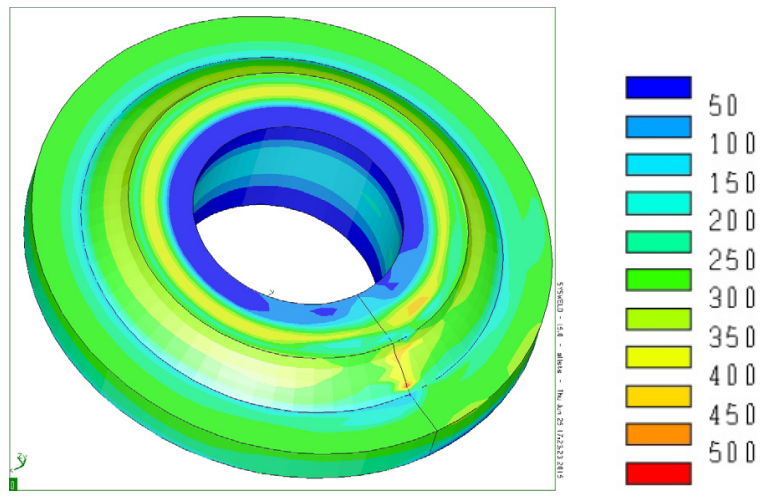

(c)

Fig. 17. Distribution of the von Mises residual stress (MPa) for the standard simulation (a) and 2 simplified simulations with 2 lengths of the current zone: $90^{\circ} \rightarrow 270^{\circ}$ (b) and $45^{\circ} \rightarrow 315^{\circ}$ (c).

allow it to cool down sufficiently. Nevertheless, the hoop stress is well computed in the current region for cases (b) and (c) as shown in Figure 19, compared to the reference case (a).

The computing times on a standard $3.4 \mathrm{GHz}$ PC with 4Go memory are given in Table 1 by using an identical time step for the time integration.

\section{Conclusion}

Welding simulations are all the more difficult as they need refined 3D models in order to capture multiphysical couplings in the HAZ but the calculation times can last several days. In this paper, a 3D simplified approach has been proposed. It consists of decreasing the computation time for the current zone of the weld. To show the 


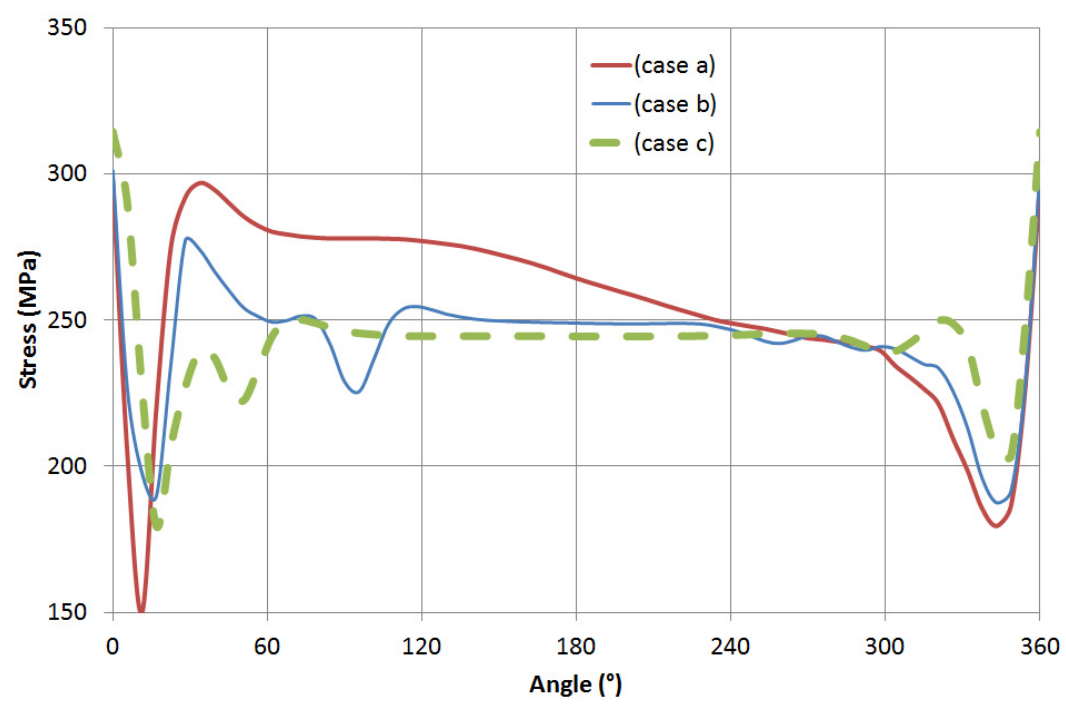

Fig. 18. Distribution of the von Mises residual stress at node $E$ (see Fig. 3).

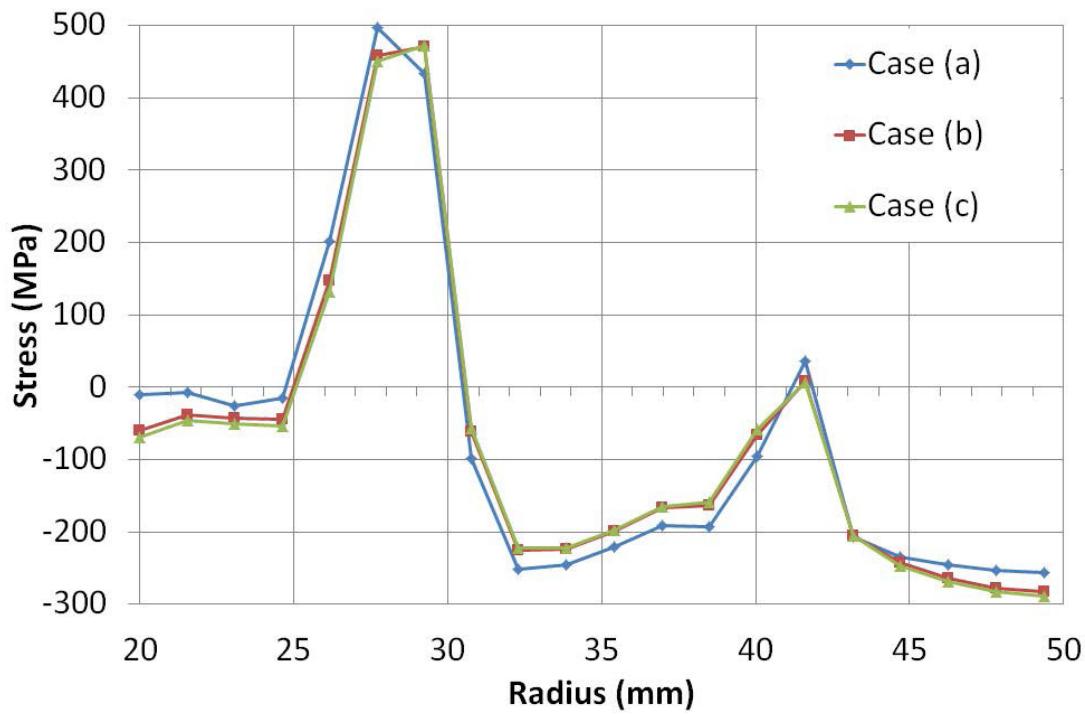

Fig. 19. Comparison of the hoop stresses (MPa) along the line $A \rightarrow B$ at $180^{\circ}$ (see Fig. 3).

Table 1. Computing times with a time step of $0.0317 \mathrm{~s}$ during heating and a greatest time step during cooling, up $100 \mathrm{~s}$.

\begin{tabular}{cccc}
\hline & Case a & Case b & Case c \\
\hline $\begin{array}{c}\text { Thermo-metallurgical } \\
\text { analysis }\end{array}$ & $2838 \mathrm{~s}$ & $2018 \mathrm{~s}$ & $1204 \mathrm{~s}$ \\
$\begin{array}{c}\text { Mechanical analysis } \\
\text { Total ratio with case a }\end{array}$ & - & $54.6 \%$ & $32.5 \%$ \\
\hline
\end{tabular}

relevance of such an approach, the methodology developed is presented through the application of a single-pass steel girth weld. For this application, the computing time can be reduced by more than $67 \%$ compared to a standard "step by step" simulation. In a general way, the gain can be estimated by the ratio of the current region length to the weld total length. It can be very significant for very long welds. This approach can be easily extended to multi-pass welding or to other continuous deposit processes such as additive manufacturing.

\section{References}

[1] E. Feulvarch, M. Fontaine, J.M. Bergheau, XFEM investigation of a crack path in residual stresses resulting from quenching, Finite Elements in Analysis and Design 75 (2013) 62-70

[2] L.E. Lindgren, H. Haggblad, J.M.A. McDill, Automatic Remeshing for Three-dimensional Finite Element Simulation of Welding, Comput. Methods Appl. Mech. Eng. 147 (1997) 401-409

[3] H. Runnemalm, S. Hyun, Three-dimensional Welding Analysis using an Adaptive Mesh Scheme, Comput. Methods Appl. Mech. Eng. 189 (2000) 515-523 
[4] P. Duranton, J. Devaux, V. Robin, P. Gilles, J.M. Bergheau, 3D modelling of multipass welding of 316L stainless steel pipe, J. Mater. Process. Technol. 153-154 (2004) 457-463

[5] M. Nastroom, L. Wikander, L.E. Karlsson, J. Goldak, Combined 3D and Shell Element Modelling of Welding, IUTAM Symp. on the Mechanical Effects of Welding, Lulea, Sweden, 1991, pp. 10-14

[6] S. Sarkani, V. Tritchkov, G. Michaelov, An efficient approach for computing residual stresses in welded joints, Finite Elements in Analysis and Design 35 (2000) 247268

[7] J. Xu, X. Jia, Y. Fan, A. Liu, C. Zhang, Residual stress analyses in a pipe welding simulation: 3D pipe versus axisymmectric models, Proc. Mater. Sci. 3 (2014) 511-516

[8] ESI Group, Users manual, 2014

[9] E. Feulvarch, V. Robin, J.M. Bergheau, Thermometallurgical and mechanical modelling of welding application to multipass dissimilar metal girth welds, Sci. Technol. Weld. Joining 16 (2011) 221-231

[10] F.M.B. Fernandes, S. Denis, A. Simon, Mathematical model coupling phase transformation and temperature evolution during quenching of steels, Mater. Sci. Technol. 1 (1985) 838-844

[11] J.-B. Leblond, J.C. Devaux, A new kinetic model for anisothermal metallurgical transformations in steel including effect of austenite grain size, Acta Metallurgica 32,1 (1984) 137-146

[12] T. Reti, Z. Freid, I. Felde, Computer simulation of steel quenching process using a multi-phase transformation model, Comput. Mater. Sci. 22 (2001) 261-278

[13] D.P. Koistinen, R.E. Marburger, A General Equation Prescribing the Extent of Austenite-Martensite Transformation in Pure Fe-C Alloys and Plain Carbon Steels, Acta Metallurgica 7 (1959) 417-426

[14] S. Denis, E. Gauthier, S. Sjostrom, A. Simon, Influence of stresses on the kinetics of pearlitic transformation during continuous cooling, Acta Metallurgica 35 (1987) 16211632
[15] J.B. Leblond, G. Mottet, J.C. Devaux, A theoretical and numerical approach to the plastic behavior of steels during phase transformation, I: Derivation of general relations, II: Study of classical plasticity for ideal-plastic phases, J. Mech. Phys. Solids 34-4 (1986) 395-432

[16] G.W. Greenwood, R.H. Johnson, The deformation of metals under small stresses during phase transformation, Proc. Roy. Soc. A 283 (1965) 403-422

[17] C.L. Magee, Transformation kinetics, microplasticity and aging of martensite in Fe-31 Ni, Ph.D. Thesis, Carnegie Institute of Technology, Pittsburgh, USA, 1966

[18] F. Abrassart, Influence des transformations martensitiques sur les propriétés mécaniques des alliages du système Fe-Ni-Cr-C, Ph.D. Thesis, University of Nancy, Nancy, 1972

[19] M. Berveiller, F.D. Fisher, Mechanics of solids with phase changes, CISM Course 368, Springer, 1997

[20] J.B. Leblond, J. Devaux, J.C. Devaux, Mathematical modelling of transformation plasticity in steels, I: Case of ideal-plastic phases, II: Coupling with strain-hardening phenomena, Int. J. Plasticity 5 (1989) 551-591

[21] H.M. Aarbogh, M. Mhamdi, A. Mo, H.G. Fjaer, Simplified method for establishing constitutive equations and flow stress data for welding stress modelling, Sci. Technol. Weld. Joining 13 (2008) 705-713

[22] Y. Vincent, J.M. Bergheau, J.B. Leblond, Viscoplastic behaviour of steels during phase transformations, Comptes Rendus Mécanique 331 (2003) 587-594

[23] J. Devaux, J.B. Leblond, J.M. Bergheau, Numerical study of the plastic behaviour of a low alloy steel during phase transformations, Journal of Shanghai Jiaotong University E-5 (2000) 206-212

[24] L. Karlsson, L.E. Lindgren, Modeling of Casting, Weld. Adv. Solid. Process. V (1991) 187-202

[25] J. Goldak, A. Chakravarti, J. Bibby, A new finite element model for welding heat sources, Metall. Mater. Trans. B 15 (1984) 299-305 